\title{
The properties of carbon stars found in the Byurakan and the Hamburg/ESO surveys ${ }^{\star, \star \star}$
}

\author{
N. Mauron ${ }^{1}$, K. S. Gigoyan ${ }^{2}$, and T. R. Kendall ${ }^{3}$ \\ ${ }^{1}$ Groupe d'Astrophysique, CNRS and Univ. de Montpellier, CC 072, Place Bataillon, 34095 Montpellier Cedex 5, France \\ e-mail: nicolas .mauron@graal.univ-montp2.fr \\ 2378433 Byurakan Astrophysical Observatory and Isaac Newton Institute of Chile, Armenian Branch, Ashtarak d-ct, Armenia \\ ${ }^{3}$ Centre for Astrophysics Research, Science and Technology Research Intitute,University of Hertfordshire, College Lane, \\ Hatfield AL10 9AB, UK
}

Received 11 July 2006 / Accepted 30 October 2006

\begin{abstract}
Aims. We analyse two samples of high latitude carbon stars found in two objective-prism surveys: the First Byurakan survey (FBS) and the Hamburg/ESO survey (HES). We determine the composition of these samples in cool AGB stars, warmer giants and dwarf carbon stars.

Methods. We list 44 new carbon stars found in the FBS and use near-infrared photometric catalogues (DENIS, 2MASS) to investigate the observational and statistical properties of the surveys. The SuperCOSMOS and USNO-B1.0 catalogues are used to study proper motions and to identify dwarfs.

Results. The colour-magnitude and colour-colour diagrams, either with visual magnitudes or with infrared data, show that the HES selects faint and relatively warm carbon stars, whereas FBS is less deep, but gives cooler objects. By comparing DENIS and 2MASS photometry, we find that HES objects with $J-K \leq 1$ are mostly non-variable giants while clear variability is often seen for $J-K \geq 1$, indicating AGB stars. By using SuperCOSMOS proper motions, 9 HES objects are found to have a proper motion $\mu$ greater than $30 \mathrm{mas} \mathrm{yr}^{-1}$ ( $3 \sigma$ detection). Five of these objects are detected at the $\geq 5 \sigma$ level. These 9 HES objects are dwarf carbon stars, including two that could belong to the halo population. The 9 carbon dwarfs are remarkably bright and deserve further study.
\end{abstract}

Key words. stars: carbon - surveys - Galaxy: halo - Galaxy: stellar content

\section{Introduction}

Carbon (C) stars are known to be of various types and origins. For example, they may be very cool and dusty giants evolving on the asymptotic giant branch (AGB) where the excess of carbon present in their atmospheres results from nucleosynthesis in the stellar interior. They can also be binary stars, often warmer giants or even dwarfs having gained carbon-rich material by accretion from a more evolved companion (for a review of $\mathrm{C}$ stars, see Wallerstein \& Knapp 1998).

The faint $\mathrm{C}$ stars located at high galactic latitude and/or far from the galactic plane are of special interest because, if they are found to be giants or AGB stars, they do not belong to the galactic disk, in contrast to the large majority of catalogued C stars. A number of methods have been used to discover these high latitude $\mathrm{C}$ stars. One method consists of selecting candidate stars through their photometric colours and performing follow-up spectroscopy. Using this method, Totten and Irwin (1998) selected very red objects in the APM photometric catalogue (from digitized Schmidt plates, Irwin 2000) over an area of $6500 \mathrm{deg}^{2}$. A similar search was carried out by

* Partly based on observations done at ESO, Chile (program 69.B0086), at Byurakan Observatory (Armenia), and at Haute Provence Observatory (France) operated by the Centre National de Recherche Scientifique.

$\star \star$ Appendices and Table 1 are only available in electronic form at http://www. aanda.org
Mauron et al. $(2004,2005)$ where candidate C stars were selected by their photometric colours in the 2MASS point-source catalogue (Skrutskie et al. 2006) and with $|b|>20^{\circ}$. Together, these works identified $\sim 130 \mathrm{cool}$ AGB C stars located in the halo and with distances up to $130 \mathrm{kpc}$. Another recent survey for faint high latitude C stars is that of Downes et al. (2004) who selected their candidates over an area of $3000 \mathrm{deg}^{2}$ by using Sloan Digital Sky Survey (SDSS) photometry. They discovered 251 relatively faint objects $(R>16)$, with a number of warm $C$ giants and at least 110 dwarf $C$ stars confirmed by their proper motions.

A very different but equally efficient method to discover C stars is to carry out objective prism spectroscopy. Early surveys designed to find $\mathrm{C}$ stars at high galactic latitude include the Case survey by Sanduleak \& Pesch (1988) covering 1000 deg ${ }^{2}$ in the north galactic cap, and the University of Michigan Thin prism Survey of MacAlpine \& Lewis (1978) covering $225 \mathrm{deg}^{2}$ in the south cap. These surveys yielded $28 \mathrm{C}$ stars (Bothun et al. 1991).

In this work, we examine the results of two more extended objective prism surveys that avoid the galactic plane. One is the First Byurakan Survey (FBS) with first results on C stars reported in Gigoyan et al. (2001; hereafter Paper I). The other is the Hamburg/ESO Survey (HES) described by Wisotzki et al. (2000). Christlieb et al. (2001) used the database of this survey for a search for stars with strong $\mathrm{C}_{2}$ and/or $\mathrm{CN}$ lines. Our goal is to investigate the properties of these two surveys, and to determine their contents in AGB stars and dwarf carbon stars. 


\section{The FBS and HES surveys}

The First Byurakan Sky Survey covers $17000 \mathrm{deg}^{2}$. According to Mickaelian et al. (2005), its magnitude limit in the $V$ band is $\sim 17.5$, but the $\mathrm{C}$ stars discovered in this survey are brighter than this limit (see Sect. 4.1). The objective prism FBS plates have a size of $4^{\circ} \times 4^{\circ}$ and a large spectral range (3400-6900 $\AA$ ). The dispersion is $2500 \AA \mathrm{mm}^{-1}$ near $\mathrm{H} \beta$ and the spectral resolution is on average $\sim 50 \AA$. For the search for C stars, this survey has been exploited so far by visual inspection. $\mathrm{C}$ stars are identified via the presence of $\mathrm{C}_{2}$ bands. From the examination of an area of $\sim 6140 \mathrm{deg}^{2}, 35 \mathrm{C}$ stars were discovered and analysed by Gigoyan et al. (2001).

The Hamburg/ESO survey for C stars covers $6400 \mathrm{deg}^{2} \mathrm{lim}$ ited by $\delta<+2.5^{\circ}$ and $|b|>30^{\circ}$. All the plates were digitized and the magnitude limit is $V \sim 16.5$. The individual spectra have a wavelength range of 3200 to $5200 \AA$ and a typical resolution of $15 \AA$ at $\mathrm{H} \gamma$. Christlieb et al. (2001) searched for C stars by applying an automated procedure to the digitized spectra, and found a total of 403 faint high latitude $\mathrm{C}$ stars in this survey.

\section{New carbon stars from the FBS}

We consider a list of 44 additional C stars found in the FBS survey (Table 1). These $44 \mathrm{C}$ stars were published by Gigoyan et al. (2006), and we give here complementary information not presented in that paper, particularly the coordinates of the objects, and a discussion of the adopted absolute magnitudes. The $44 \mathrm{C}$ stars listed in Table 1 were found in regions of the sky which had not been inspected previously. The table in Appendix A provides the characteristics of the zones that were examined and their areas. The total area of these zones is $9755 \mathrm{deg}^{2}$. Slit spectroscopy of FBS C stars has been performed to confirm their carbon-rich nature. All observed objects were found to be carbon rich. Details of the instrumental configuration, as well as figures of the spectra obtained, can be found in the online Appendix. Two representative low resolution spectra are shown in Fig. 1.

Almost all stars classified as N-type from the FBS plate spectra or from the follow-up spectroscopy were found to have a 2MASS colour of $J-K>1.4$. The only exception is one object (\#68). For these red stars, distances in Col. 10 of Table 1 were estimated from their $K$ magnitude and $J-K$ colour index. For a given $J-K$ index, an average absolute $K$-band magnitude was derived by considering LMC C stars as templates, and by assuming a distance modulus $m-M=18.50$ for the LMC. These absolute magnitudes are found to be typically between -7.4 and -7.9 . The dispersion of these values is of the order of $0.4-0.5 \mathrm{mag}$.

Concerning the $\mathrm{C}$ stars that are not N-type, the $J-K$ index typically lies between 0.6 and 1.0. For these colours, the scatter of $K$ magnitudes for C stars in the LMC is too large to be used as a luminosity indicator of these stars. The low-resolution slit spectra that have a sufficiently good signal-to-noise ratio show that, in many cases, there is a strong $\mathrm{CH}$-band at $4300 \AA$. In principle, these objects could be $\mathrm{CH}$-type $\mathrm{C}$ stars. In the absence of data concerning their metal abundances, proper motions and radial velocities, we cannot exclude that some objects are R-type. The spectroscopic differences between these two classifications can be very subtle and practically invisible in our spectra (see for example Fig. 2 of Goswami 2005). Here, we make the simplifying hypothesis that all stars with $J-K<1.2$ are CH-type.

In their Table 4, Totten and Irwin (1998) take $M_{R}=-3.5$ for both $\mathrm{N}$-type and $\mathrm{CH}$-type stars, but they note that $\mathrm{CH}$-type are
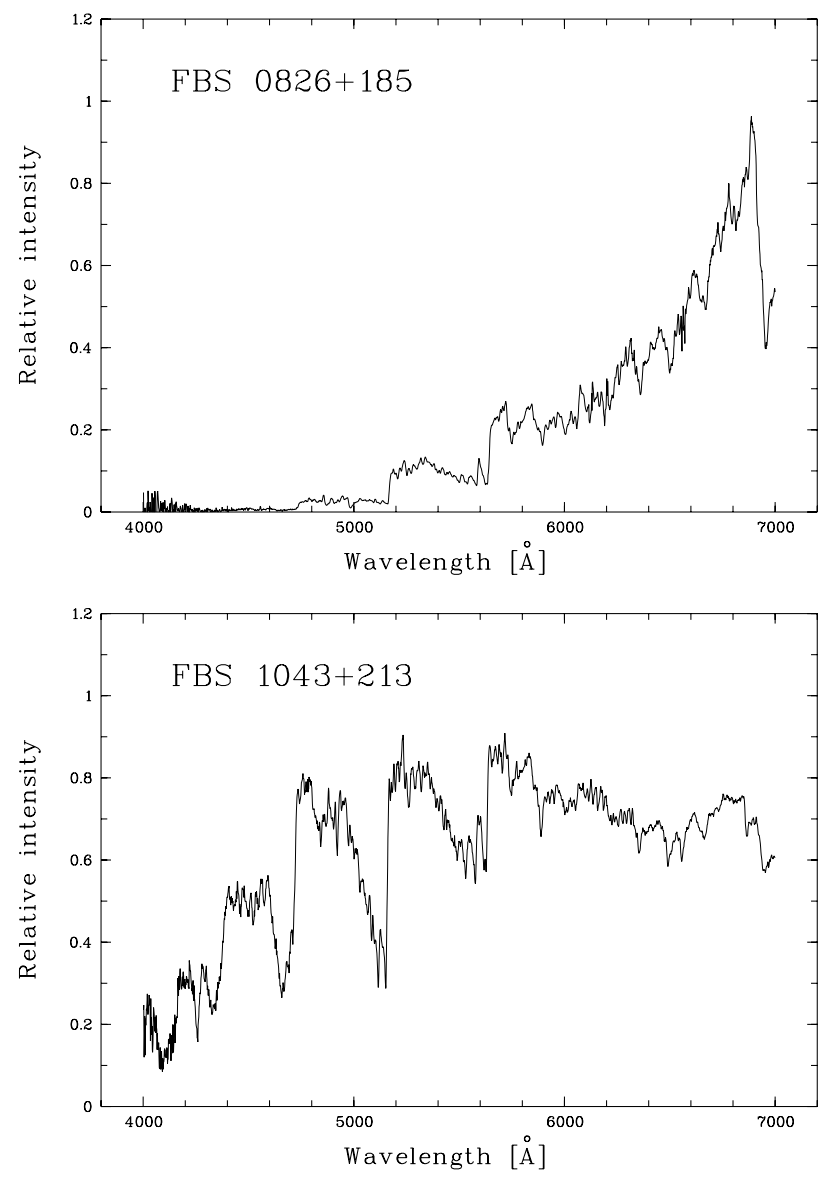

Fig. 1. Two representative low resolution spectra obtained at Byurakan Observatory. In the upper panel, the object is FBS $0826+185$, a cool Ntype star with $R=11.5, B-R=4.6, J-K=1.68$ and $b=+30^{\circ}$. In the lower panel, the object is FBS $1043+213$, a warm C star with $R=11.2$, $B-R=2.3, J-K=0.88$ and $b=+61^{\circ}$.

intrinsically somewhat fainter. In fact, $\mathrm{CH}$ stars have a range of luminosities, from $M_{V} \sim-2$ at the top of the red giant branch like the $\mathrm{CH}$ stars in $\omega$ Centauri, down to $M_{V} \sim+4$ on the subgiant branch (Wallerstein \& Knapp 1998). A high luminosity will be assumed here. As the mean colour $V-R$ of this kind of high latitude warm $\mathrm{C}$ star is $\sim 0.6(\sigma=0.5)$, as measured for the HES stars, we adopted $M_{R}=-2.5$. Distances quoted in Table 1 were derived after taking into account interstellar extinction derived from the maps of Schlegel et al. (1998).

There are large uncertainties in these distances, both for $\mathrm{N}$-type and other objects, at least $\pm 25 \%$. It can be seen that $\mathrm{N}$-type stars (sources with $J-K>1.2$ ) have distances up to $16 \mathrm{kpc}$ from the Sun, and heights from the galactic plane of up to $12 \mathrm{kpc}$. For warmer objects, the range in distance is slightly lower $(13 \mathrm{kpc})$, provided our choice of absolute magnitude is correct.

\section{Properties of the FBS and HES samples}

\subsection{Visible photometry}

Here, we examine the R-band magnitude and $B-R$ colour index for the 79 FBS $C$ stars, and make a comparison with the $C$ stars from the HES survey. These $R$ and $B$ magnitudes are from the USNO-A2.0 catalogue in the great majority of cases. The accuracy of this visible photometry is of the order of $\sim 0.4 \mathrm{mag}$. 

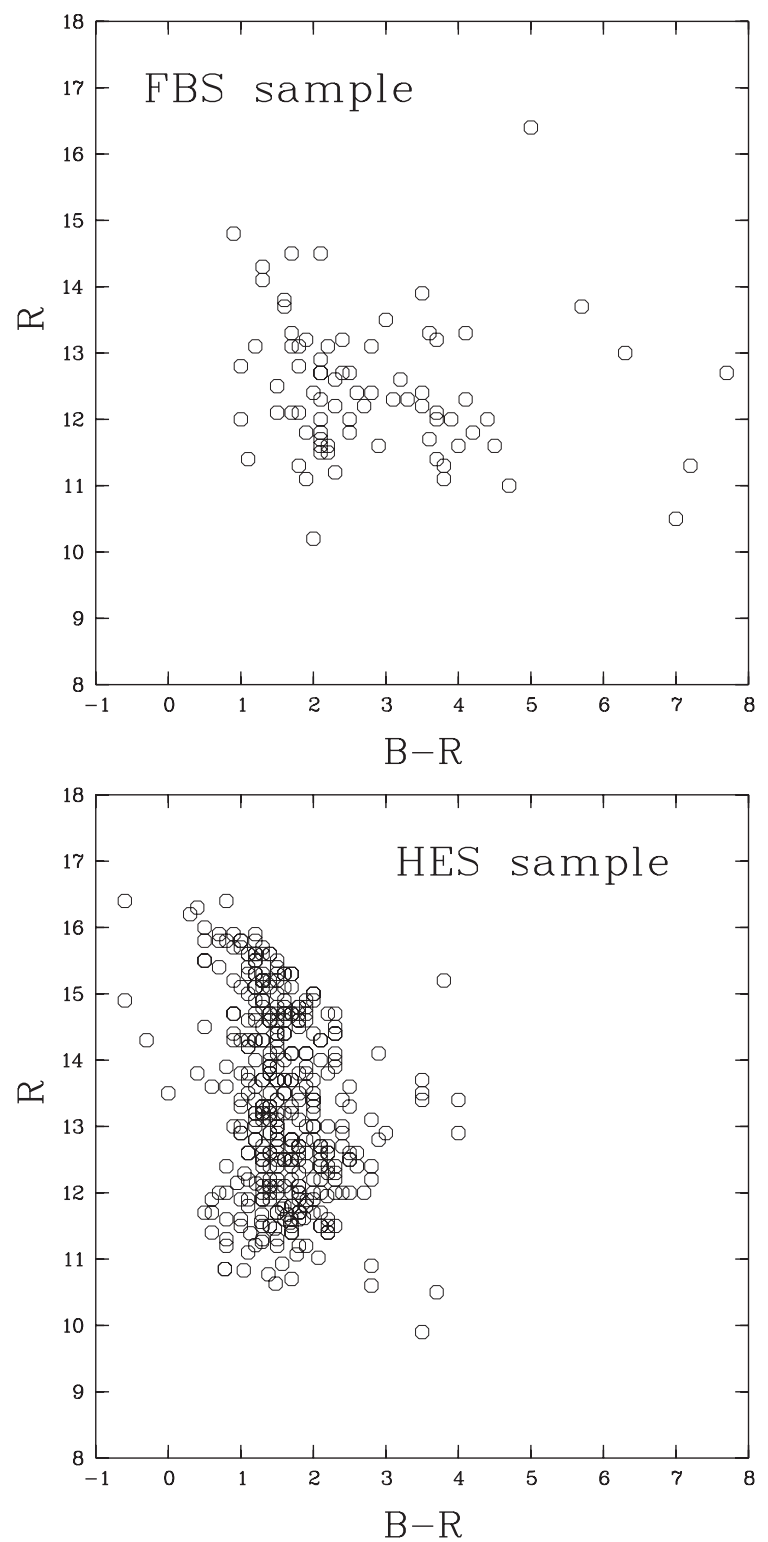

Fig. 2. Plots of the $R$-band magnitude as a function of the $B-R$ colour index. Upper panel: FBS objects from Paper I and this paper. Lower panel: HES objects.

In Fig. 2, upper panel, one can see that most of the 79 FBS stars have $R$ between 11 and 14.5. One star has $R=16.4$. For this star, FBS $1502+359$ (Paper I), $R$ is from the APM catalogue because no USNO-A2.0 $R$ or $B$ magnitude was available. This very red object may be a variable observed at a magnitude brighter than $R=16$ when the FBS plate was exposed. Therefore, the faintest $R$ magnitude for FBS stars appears to be $R \sim 14.5$. The FBS stars range from $B-R \sim 1$ up to $B-R \sim 6-7$, i.e., very red stars can be detected.

The lower panel of Fig. 2 presents the same $R$ vs. $B-R$ diagram for the HES survey. Most of the stars have $R$ between 11 and 16, showing that the HES survey is about 1.5 mag deeper in $R$ than the FBS. For example, it is found that in the FBS sample $7 \%$ have $R>14$, compared with $33 \%$ for the HES survey. Concerning the colours, one sees that very few HES stars have $B-R>3$ ( $2 \%$ of the sample); the corresponding number is $32 \%$ for the FBS.

Figure 2 also shows that, for a given magnitude range, common to both HES and FBS (for example $R$ between 12 and 13 ), there is a considerably larger number of sources detected in the HES than in the FBS. This is not due to a limiting magnitude effect, but more probably to the superiority of automatic detection of carbon features compared to visual inspection. The densities of detected sources per square degree are $\sim 0.06$ for HES and only $\sim 0.005$ for FBS. The FBS plates have been digitized (Mickaelian et al. 2005; see also the web site http: //astro1.phys . uniroma.it/DFBS/fbs.htm) and an automated search and classification of fainter $\mathrm{C}$ stars in the FBS should be feasible in the future.

\subsection{MASS photometry}

Photometry from 2MASS is available for all sources in our two samples (except one object in the HES). One advantage of 2MASS compared to USNO-A2.0 is its much greater photometric accuracy. For FBS sources, the errors on $J$ and $K$ are all less than 0.03 mag, except in one case. For HES sources, which can be fainter in $J$ and $K$, the errors on $J$ are lower than 0.04 mag for $98 \%$ of objects, and the error in $K$ is lower than 0.06 mag for $98 \%$ of objects. A few cases, with $K$ fainter than 14, have lower accuracies, although they are always smaller than 0.1 mag.

In the colour-magnitude diagram for the HES stars (Fig. 3, lower panel), one sees two populations, one with $J-K<1$ that we interpret as warm (giant or dwarf) $\mathrm{C}$ stars, and a second one with $J-K>1$. This last group comprises 29 objects (7\% of the total), and we suggest that it is composed of optical (i.e., not very dusty) AGB C stars.

There are a few especially distant AGB stars in this last group, since there are 8 objects with $J-K>1$ and $K \geq 10$. All lie at galactic latitude $|b|>30^{\circ}$. If one selects objects with $J-K>1.2$ and $K>10$, we find 3 objects. Two objects, HE 0207-0211 and HE 1442-0058, with distances 30 and $40 \mathrm{kpc}$, were already known and listed in Totten and Irwin (1998). The third, HE 1344-0411, was unknown. It has the following characteristics: $K=10.47, J-K=1.24, b=+55^{\circ}$. Based on the $K$-band luminosity of the LMC C stars having the same $J-K$, its absolute magnitude is $M_{K} \approx-7.0$. Consequently, its distance is about $30 \mathrm{kpc}$ and its height above the galactic plane is $\sim 25 \mathrm{kpc}$.

Figure 3, upper panel, shows the colour-magnitude diagram for FBS stars. There are 36 objects with $J-K>1$, representing $46 \%$ of the total sample. In the FBS sample, none of the stars with $J-K>1$ reaches $K=10$ because the FBS survey is poorer in depth than the HES and misses C stars which are too faint. However, the FBS is able to detect very red stars with $J-K>2$, while none are detected by the HES.

Figure 4 shows colour-colour diagrams with 2MASS data. The diagram of the FBS sample shows the three families of C stars that FBS can detect, i.e. warm C stars, optical AGB C stars with $J-K$ between 1 and 2, and very red AGB C stars with $J-K>2$. The HES experiment did not detect any very red C stars. This is obviously due to its blue-visible spectral range. For example, HES missed $11 \mathrm{C}$ stars listed in Mauron et al. (2004, 2005), although these objects satisfy $R<16, b<-30^{\circ}$ and $\delta<+2^{\circ}$. The reason is that their $B-R$ colour is too large and the signal at blue wavelengths is too faint.

\subsection{Variability}

Because the HES objects lie at $\delta<+2.5^{\circ}$, a significant fraction of them were seen in the DENIS southern infrared survey (Epchtein 1998; Epchtein et al. 1999). From the last DENIS release available at the CDS (Strasbourg), we found that of 

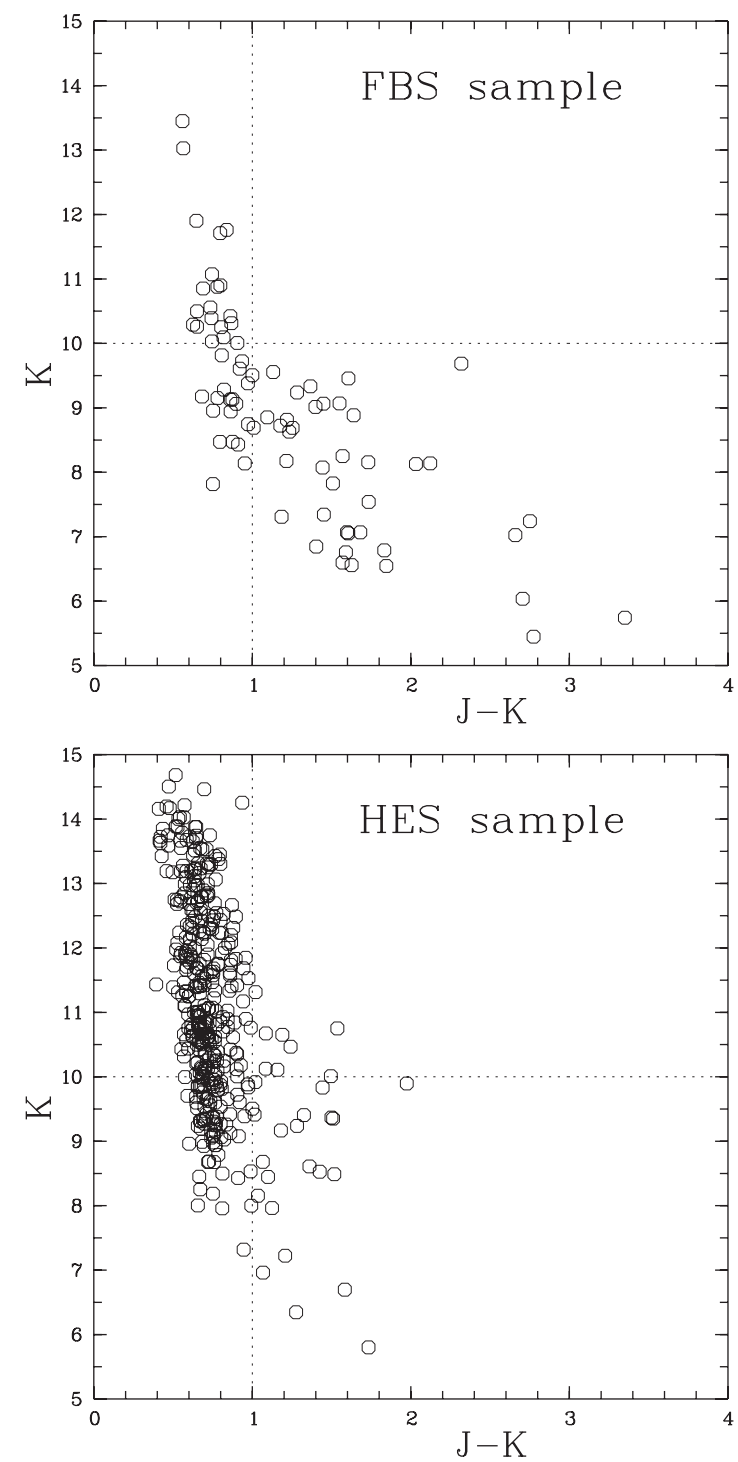

Fig. 3. Colour-magnitude diagrams $K$ vs. $J-K$. Lower panel: HES sources. Upper panel: FBS sources. In both panels, the limits $J-K=1$ and $K=10$ are overplotted (see text).

402 HES objects, 271 have DENIS data. The DENIS survey provides $I, J, K$-band photometry, so that one can compare Denis $J$ and $K$ with the 2MASS $J$ and $K$ observations in order to detect photometric variability. A direct comparison can be made because the transmission functions of these filters are very similar (see Fig. 4 of Bessel 2005), and because a quantitative analysis demonstrated no significant differences between the two photometries (Cabrera-Lavers \& Garzon 2003, and references therein). The epoch differences between 2MASS and DENIS observations are scattered in the range 0 to 1500 days. In general, the measurement uncertainty on $K$ is larger than in $J$, so we limit our investigation to the $J$-band.

Figure 5 shows the difference $\Delta J=J_{\text {DENIS }}-J_{2 M A S S}$ as a function of the 2MASS $J-K$ colour. It can be seen that for $J-K<1$, the majority of the objects have $\Delta J$ close to zero. There is only one object at $J-K=0.71$ for which $\Delta J=0.63$, and this variation in $J$ is supported by a similar although smaller variation in $K(\Delta K=0.37)$. If we omit this variable object, for a subsample of 245 objects, the average of $\Delta J$ is $0.005 \mathrm{mag}$, with an rms of $0.078 \mathrm{mag}$. Given the uncertainties on the DENIS and 2MASS photometry, a very large majority of stars in this
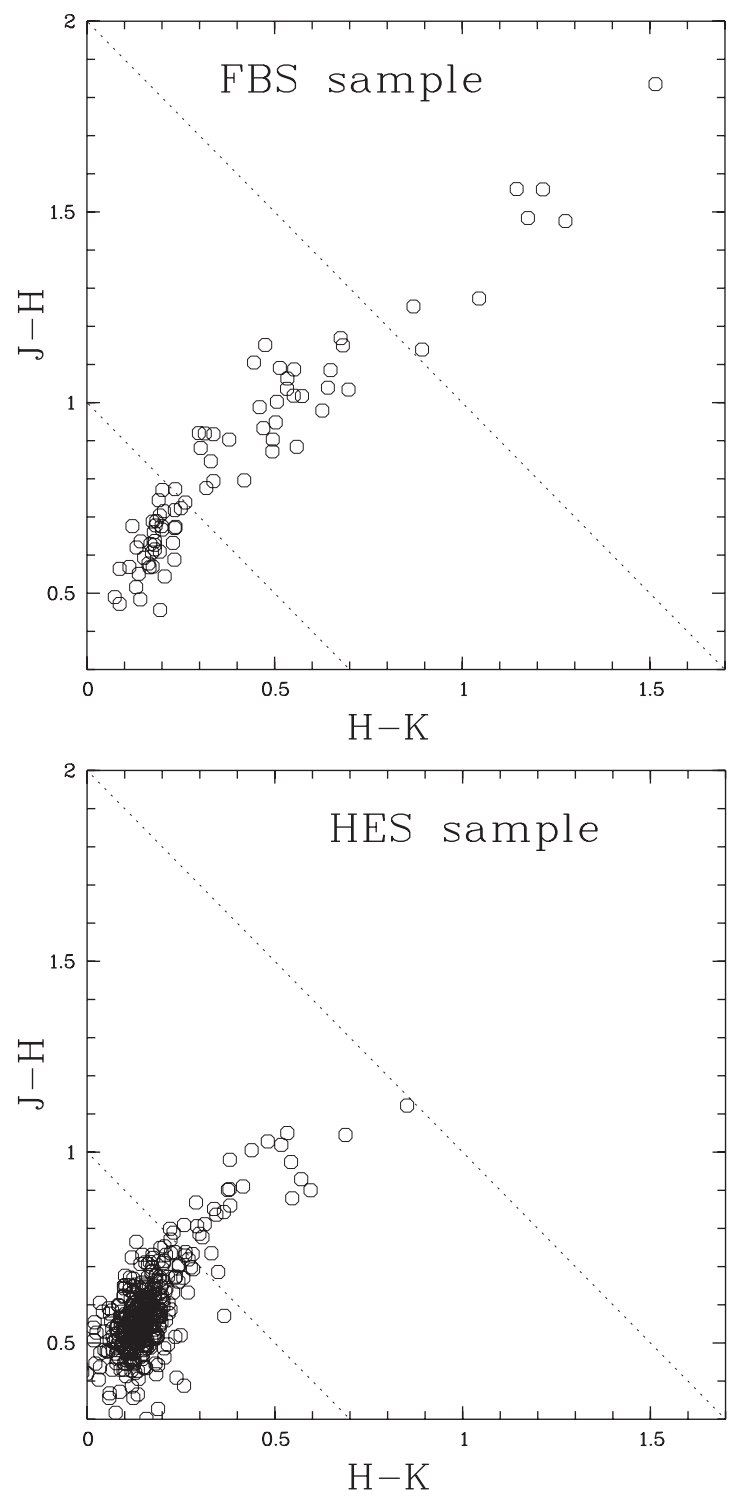

Fig. 4. Colour-colour diagrams with $J-H$ vs. $H-K$ (from 2MASS photometry). Lower panel: HES sources. Upper panel: FBS sources. In both panels, the dotted lines correspond to $J-K=1$ and $J-K=2$.

subsample are thus stable in flux. In contrast, for the subsample formed by the 22 redder stars with $J-K>1, \sim 8$ stars display variability, of up to about one magnitude. For these 22 objects, the average $\Delta J$ is -0.10 with a dispersion of 0.34 mag.

Concerning the FBS survey (which covers only a fraction of the southern hemisphere), we found only 15 stars belonging to the DENIS survey, so that the statistics are poor. Only one source shows a significant flux variation between DENIS and 2MASS data. It is FBS 0846-071 $\left(\alpha=08^{\mathrm{h}} 49^{\mathrm{m}} 10.96^{\mathrm{s}}\right.$, $\left.\delta=-7^{\circ} 21^{\prime} 44.24^{\prime \prime}(\mathrm{J} 2000)\right)$, with $R=13.5$ and $B-R=3.0$ (see Paper I). Its variation is: $J_{\text {DENIS }}-J_{2 \text { MASS }}=2.33$, and $K_{\text {DENIS }}-K_{2 \text { MASS }}=1.99$. This star is a confirmed AGB C star with a distance of $\sim 17 \mathrm{kpc}$ and a height from the galactic plane of $\sim 6 \mathrm{kpc}$.

\subsection{Objects with proper motion}

According to Christlieb et al. (2001), the HES sample may contain a small proportion of dwarf $\mathrm{C}$ stars. To investigate this point, we have searched for counterparts of the HES objects 


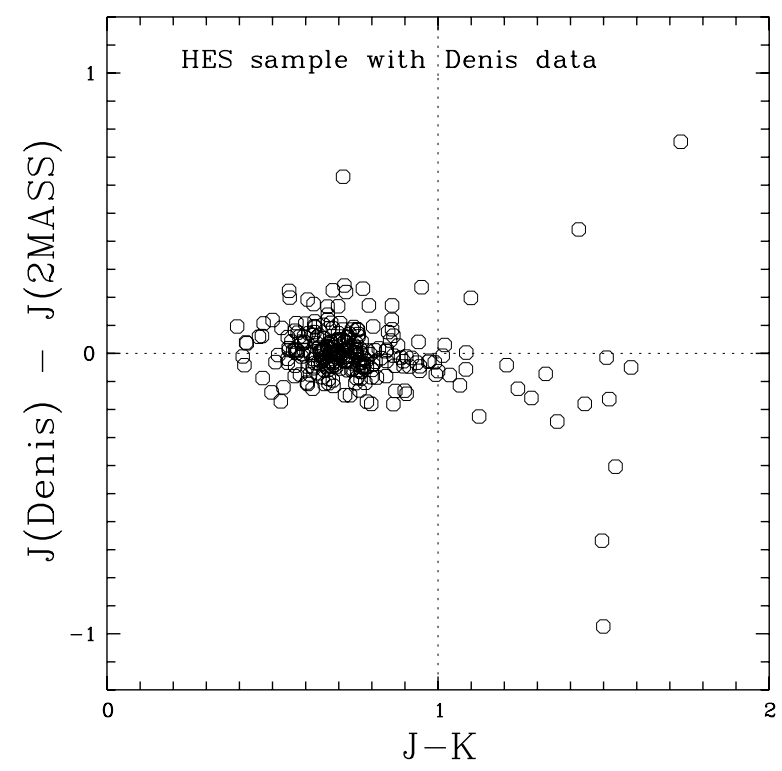

Fig. 5. Difference between J(DENIS) and J(2MASS) plotted as a function of the $J-K$ colour index of 2MASS.

in the SuperCOSMOS catalogue (Hambly et al. 2001a-c). SuperCOSMOS provides proper motions and their errors $\sigma$ for the Southern hemisphere. These errors are of the order of 6 to $13 \mathrm{mas} \mathrm{yr}^{-1}$. A total of $402 \mathrm{HES}$ objects were found in SuperCOSMOS. Figure 6 shows $\mu_{\delta}$ as a function of $\mu_{\alpha} \cos (\delta)$. Crosses have been overplotted when two conditions are met: the

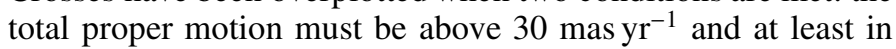
$\alpha$ or in $\delta$ one finds a $\geq 3 \sigma$ detection. One sees that 9 sources satisfy these conditions and Table 2 provides the details of the SuperCOSMOS data. Table 2 also provides distances and tangential velocities, which will be discussed in the next section.

We have also searched for FBS objects with proper motions.

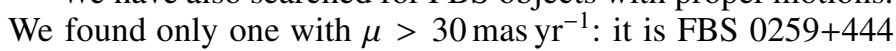
(listed in Paper I). The USNO-B1.0 catalogue of Monet et al.

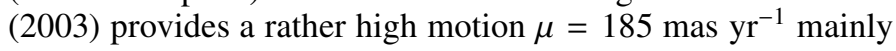
in $\alpha$. However, comparison of POSS images does not show any corresponding displacement, of the order of $\sim 7^{\prime \prime}$, for an interval of 38 years between examined plates. We noted instead that the star is blended with a fainter companion to the East, suggesting that the motion is an artefact.

\section{Discussion}

The accuracy of 2MASS photometry has allowed a clear separation of the HES objects into two groups. The objects with $J-K>1$ are presumably AGB stars, an interpretation supported by the high fraction of variable stars in this group. There are no such objects with $K>11$ because AGB stars as faint as this in $K$ (and consequently very distant in the halo) are very rare, and are only found with specific methods (see, e.g. Mauron et al. 2004, 2005). We suggest that HES objects with $J-K<1$ are red giant branch objects, or for a small fraction, dwarfs (see below). The giants are likely ordinary early R-type stars or CH-type C stars but high resolution spectroscopy is needed to separate the two types with certainty. Goswami (2005) obtained medium resolution $(\lambda / \delta \lambda \sim 1000)$ spectra of 91 HES stars, and concluded the presence of 35 "potential candidate" $\mathrm{CH}$ stars.

On the $(K, J-K)$ colour-magnitude diagram for HES objects, there is a clear tendency towards bluer $J-K$ colours with progressively fainter $K$ magnitudes. At $K=9, J-K$ is about 0.8 , but at $K=14, J-K$ is about 0.5 (Fig. 3). This trend is also visible for the FBS stars, although the statistics are much poorer. Joyce (1998) presented near-infrared spectroscopy of faint high latitude C (FHLC) stars. His spectra show that the $J, H$, and $K$ bandpasses are dominated by $\mathrm{CN}, \mathrm{C}_{2}$ and $\mathrm{CO}$ molecular bands. While these bands are very strong for AGB stars with $J-K>1$, they can nearly disappear for some FHLCs like CLS 26 and CLS 80 for which $J-K \approx 0.50$ (Joyce 1998). In that case the spectra are close to a smooth continuum decreasing with $\lambda$. Therefore, we suggest that this colour-magnitude trend indicates that bright objects have more pronounced molecular bands in $J H K$ than faint objects. A possible explanation is that faint objects are on average more distant and probe populations (thick disk, halo) where the mean metallicity is lower, the giants on average hotter, and consequently the molecular bands fainter.

The fact that a few (9) HES sources have measurable proper motions is most noteworthy. Christlieb et al. (2001) had predicted that 10-5 out of the 403 HES objects should be dwarf carbon stars (dCs), so that the agreement between their predictions and our findings is reasonable. A remarkable property of the HES dCs is their high apparent intensity. All are brighter than $R=15$ and 5 are brighter than $R=14$. These $\mathrm{dCs}$ are clearly brighter than those from the SDSS survey (Downes et al. 2004). In Table 2, we have also listed the $K$ magnitude in order to compare our sample with the $\mathrm{C}$ dwarfs listed by Lowrance et al. (2003). These authors give in their Table 1 a list of all $\mathrm{C}$ dwarfs known at the time of their writing, and 5 of their objects have $K<12.5$. In our sample, we also find 5 sources with $K<12.5$, i.e. we double the number of bright $\mathrm{dC}$ targets especially suited to spectroscopic investigations.

In Table 2, we have also estimated distances for each C dwarf by adopting a $K$-band absolute magnitude $M_{K}=+6.3$. This $M_{K}$ comes from the only three $\mathrm{C}$ dwarfs for which a parallax has been measured (see Table 1 of Lowrance et al. 2003, Note d). Distance estimates are in the range 100 to $\sim 500 \mathrm{pc}$. This allows us to calculate the tangential velocity $v_{\mathrm{t}}$ in $\mathrm{km} \mathrm{s}^{-1}$. Most of our carbon dwarfs have $v_{\mathrm{t}}$ between 20 and $60 \mathrm{~km} \mathrm{~s}^{-1}$, but two have a $v_{\mathrm{t}}$ of $120 \mathrm{~km} \mathrm{~s}^{-1}$. If our distance estimates are correct, these two objects could be halo carbon dwarfs.

\section{Conclusions}

In this paper on carbon stars located far from the galactic plane, we have considered two objective-prism surveys and analysed their properties. Our main results are the following:

- A list of 44 new C stars in the FBS has been considered. Their distances from the Sun, and from the galactic plane, are up to $\sim 15 \mathrm{kpc}$.

- The 2MASS colour-magnitude and colour-colour diagrams show that the HES contains at least two populations, one bluer and one redder than $J-K=1$. The FBS can detect stars with very red colours $(J-K>2)$.

- When one compares 2MASS and DENIS data, the HES blue population shows no sign of variability, while the red one does. The red population is probably made of AGB stars, while the blue population contains mainly stars on the red giant branch.

- In the blue population, the faint stars have a mean $J-K$ lower than the bright stars. We propose that, on average, these faint stars come from more distant populations of lower metallicity (thick disc, halo) than the bright stars. At low metallicity, the giants are warmer, and the molecular bands in the $J H K$ 
Table 2. HES objects with significant proper motions in SuperCOSMOS. In the 3rd and 4th columns, the proper motions in $\alpha$ and $\delta$, and their respective $1 \sigma$ errors are given, in mas $\mathrm{yr}^{-1}$. In Cols. (5) and (6), we have listed the ratios $\kappa_{1}$ and $\kappa_{2}$ between measurements and their errors. For at least one coordinate, these ratios $\kappa$ have to be greater than 3. Column (7) is an $R$-band magnitude from SuperCOSMOS. Col. (8) is the $K$-magnitude from 2MASS. Col. (9) is the total proper motion in mas $\mathrm{yr}^{-1}$. Column (10) is the distance in pc. Column (11) is the tangential velocity in $\mathrm{km} \mathrm{s}^{-1}$.

\begin{tabular}{|c|c|c|c|c|c|c|c|c|c|c|}
\hline Name & Coordinates (J2000) & $\mu_{\alpha} \cos (\delta)$ & $\mu_{\delta}$ & $\kappa_{1}$ & $\kappa_{2}$ & $R$ & $K$ & $\mu$ & $d$ & $v_{\mathrm{t}}$ \\
\hline (1) & (2) & (3) & (4) & (5) & (6) & (7) & (8) & (9) & (10) & (11) \\
\hline HE 0009-1824 & $0012 \quad 18.5-180755$ & $+33.9 \pm 10.0$ & $-17.5 \pm 11.0$ & 3.4 & 1.6 & 15.5 & 13.66 & 38 & 300 & 54 \\
\hline HE $0343-5714$ & $034411.8-570509$ & $+38.3 \pm 7.2$ & $-41.9 \pm 5.9$ & 5.3 & 7.1 & 15.9 & 14.68 & 57 & 475 & 128 \\
\hline HE $0432-3128$ & $043406.7-312211$ & $+86.5 \pm 4.6$ & $+39.1 \pm 5.4$ & 18.8 & 7.3 & 15.4 & 13.51 & 95 & 280 & 125 \\
\hline HE 0930-0018 & $093324.6-003145$ & $-62.1 \pm 6.0$ & $+37.5 \pm 5.9$ & 10.3 & 6.4 & 13.7 & 11.33 & 73 & 100 & 34 \\
\hline HE $1058-2228$ & $110121.8-224431$ & $+5.7 \pm 10.1$ & $-46.9 \pm 13.9$ & 0.6 & 3.4 & 12.4 & 11.39 & 47 & 100 & 22 \\
\hline HE $1116-1628$ & $111903.9-164449$ & $-23.5 \pm 6.7$ & $+29.8 \pm 4.6$ & 3.5 & 6.5 & 14.4 & 12.48 & 38 & 170 & 31 \\
\hline HE 1205-0417 & $120751.7-043440$ & $-15.2 \pm 6.5$ & $-70.0 \pm 6.1$ & 2.3 & 11.5 & 14.2 & 11.68 & 72 & 120 & 40 \\
\hline HE 1358-2508 & $140154.9-285624$ & $+43.8 \pm 9.0$ & $+1.0 \pm 8.2$ & 4.9 & 0.1 & 15.8 & 13.44 & 44 & 270 & 56 \\
\hline HE 2329-0716 & $233154.9-065932$ & $+54.2 \pm 12.7$ & $-3.7 \pm 14.7$ & 4.3 & 0.2 & 13.9 & 12.16 & 54 & 150 & 39 \\
\hline
\end{tabular}

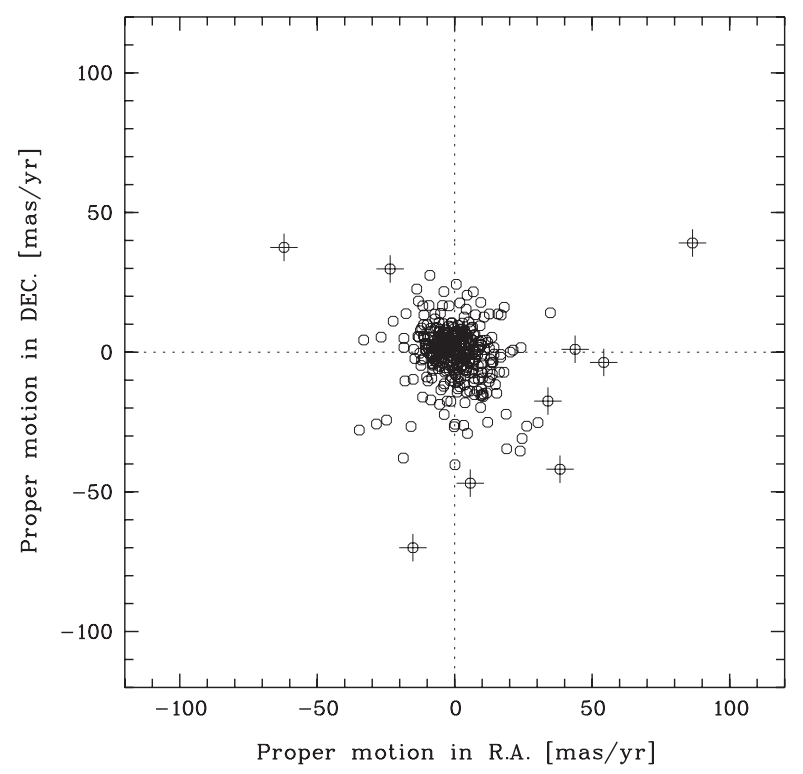

Fig. 6. Proper motions of 402 HES objects, with $\mu_{\alpha} \cos (\delta)$ in abscissa and $\mu_{\delta}$ in ordinates. Circles with an overplotted cross correspond to

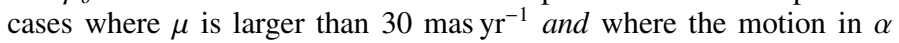
or $\delta$ is detected with at least a $3 \sigma$ quality.

filters are much less pronounced, and consequently the $J-K$ index becomes smaller.

- We investigated the proper motions of the HES stars by using the SuperCOSMOS data. We found 9 cases having detections at more than the $3 \sigma$ level in $\alpha$ or $\delta$, and with $\mu>30$ mas $\mathrm{yr}^{-1}$. These $\mathrm{C}$ dwarfs have an apparent brightness comparable to the brightest such cases presently known, and two objects could be halo C dwarfs.
Acknowledgements. We thank our referee, N. Christlieb, for several useful remarks. We also thank the people and organizations that were involved in the construction of the unique astronomical catalogues that were used in this research, particularly 2MASS, DENIS, USNO-B1 and SuperCOSMOS.

\section{References}

Bessel, M. S. 2005, ARA\&A, 43, 293

Bothun, G. L., Elias J. H., MacAlpine, G., et al. 1991, AJ, 101, 2220

Cabrera-Lavers, A., \& Garzon, F. 2003, A\&A, 403, 383

Christlieb, N., Green, P. J., Wisotzki, L., \& Reimers, D. 2001, A\&A, 375, 366

Downes, R. A., Margon, B., Anderson, S. F., et al. 2004, AJ, 127, 2838

Epchtein, N. 1998, The impact of near-infrared surveys on galactic and extragalctic astronomy. Proc. 3rd. Euroconf., Kluwer ASSL, 230

Epchtein, N., Deul, E., Derriere, S., et al. 1999, A\&A, 349, 236

Gigoyan, K., Mauron, N., Azzopardi, M., et al. 2001, A\&A, 371, 560 (Paper I)

Gigoyan, K., Mickaelian, A. M., Mauron, N. 2006, Astrofizica, 49, 197

Goswami, A. 2005, MNRAS, 359, 531

Hambly, N. C., Mac Gillivray, H. T., Read, M. A., et al. 2001a, MNRAS, 326, 1279

Hambly, N. C., Irwin, M. J., \& Mac Gillivray, H. T. 2001b, MNRAS, 326, 1295

Hambly, N. C., Davenhall, A. C., Irwin, M. J., \& Mac Gillivray, H. T. 2001c, MNRAS, 326, 1315

Irwin M. J. 2000, The APM Catalogue (the web site is http://www.ast.cam.ac.uk/ apmcat/)

Joyce, R. R. 1998, AJ, 115, 2059

Lowrance, P. J., Kirkpatrick, J. D., Reid, I. N., et al. 2003, ApJ, 584, L95

MacAlpine, G. M., \& Lewis, D. 1978, ApJS, 36, 587

Mauron, N., Azzopardi, M., Gigoyan, K., \& Kendall, T. R. 2004, A\&A, 418, 77

Mauron, N., Kendall, T. R., \& Gigoyan, K. 2005, A\&A, 438, 867

Mickaelian, A. M., Hagen, H.-J., Sargsyan, L. A., et al. 2005, CDS cata$\log \mathrm{VI} / 116$, Byurakan Observatory Publications (see also the web site http://astro1.phys. uniroma1.it/DFBS/fbs.htm)

Monet, D. G., Levine, S. E., Canzian, B., et al. 2003, AJ, 125, 984

Sanduleak, N., \& Pesch P. 1988, ApJS, 66, 387

Schlegel, D. J., Finkbeiner, D. P., \& Davis M. 1998, ApJ, 500, 525

Skrutskie, M. F., Cutri, R. M., Stiening, R., et al. 2006, AJ, 131, 1163

Totten, E. J., \& Irwin M. J. 1998, MNRAS, 294, 1

Wallerstein, G., \& Knapp, G. R. 1998, ARA\&A 36, 369

Wisotzki, L., Christlieb, N., Bade, N., et al. 2000, A\&A, 358, 77 


\section{Online Material}


N. Mauron et al.: FBS and HES carbon stars, Online Material $p 2$

Table 1. New FBS carbon stars. Column 1: Running number following that given in Gigoyan et al. (2001). Column 2: FBS name. Columns 3 and 4: J2000 coordinates from 2MASS; Col. 5: Galactic coordinates; Cols. 6 and 7: $R$ mag. and $B-R$ colour from the USNOC-A2.0 catalogue; Cols. 8 and 9: $K$ mag and $J-K$ colour from 2MASS; Col. 10: distance estimate in kpc; Col. 11: estimate of the height in kpc above or below the galactic plane.

\begin{tabular}{|c|c|c|c|c|c|c|c|c|c|c|}
\hline (1) & (2) & (3) & (4) & (5) & (6) & (7) & (8) & (9) & (10) & (11) \\
\hline No. & FBS No. & $\begin{array}{c}\alpha(\mathbf{J}) \\
\mathrm{h} \mathrm{m} \mathrm{s}\end{array}$ & $\begin{array}{l}\delta(\mathrm{J}) \\
\circ,\end{array}$ & $l, b$ & $\begin{array}{c}R \\
\mathrm{mag}\end{array}$ & $\begin{array}{r}B-R \\
\text { mag }\end{array}$ & $\begin{array}{r}K \\
\text { mag }\end{array}$ & $\begin{array}{r}J-K \\
\text { mag }\end{array}$ & $\begin{array}{r}d \\
\mathrm{kpc}\end{array}$ & $\begin{array}{r}z \\
\mathrm{kpc}\end{array}$ \\
\hline 36 & $0318+238$ & 032116.3 & +235850 & $161-27$ & 10.2 & 2.0 & 7.82 & 0.75 & 2.5 & -1.1 \\
\hline 37 & $0502+088$ & 050500.2 & +085609 & $191-18$ & 12.7 & 7.7 & 6.04 & 2.71 & 5.0 & -1.5 \\
\hline 38 & $0520+029$ & 052302.3 & +030145 & $199-18$ & 12.3 & 3.3 & 6.76 & 1.59 & 8.0 & -2.5 \\
\hline 39 & $0707+270$ & 071047.9 & +265903 & $190+16$ & 11.3 & 7.2 & 5.45 & 2.77 & 4.0 & +1.1 \\
\hline 40 & $0707+310$ & 071048.3 & +305546 & $186+17$ & 11.0 & 4.7 & 6.60 & 1.57 & 7.5 & +2.0 \\
\hline 41 & $0729+269$ & 073232.7 & +264715 & $192+20$ & 11.7 & 3.6 & 8.16 & 1.73 & 16.0 & +5.5 \\
\hline 42 & $0731+274$ & 073423.9 & +271912 & $192+21$ & 12.0 & 4.4 & 7.54 & 1.73 & 12.0 & +4.5 \\
\hline 43 & $0826+185$ & 082915.1 & +182307 & $206+30$ & 11.5 & 4.6 & 7.07 & 1.68 & 10.0 & +5.0 \\
\hline 44 & $0826+109$ & 082929.0 & +104624 & $214+27$ & 13.0 & 6.3 & 8.14 & 2.12 & 16.0 & +7.5 \\
\hline 45 & $0900+034$ & 090315.7 & +031402 & $226+31$ & 12.1 & 1.7 & 9.13 & 0.88 & 8.0 & +4.0 \\
\hline 46 & $0904+213$ & 090712.0 & +210853 & $206+39$ & 11.6 & 2.1 & 8.96 & 0.75 & 6.5 & +4.0 \\
\hline 47 & $0916+029$ & 091850.8 & +024504 & $229+33$ & 11.4 & 1.1 & 8.47 & 0.80 & 6.0 & +3.0 \\
\hline 48 & $1043+213$ & 104605.9 & +210227 & $218+61$ & 11.2 & 2.3 & 8.47 & 0.88 & 5.5 & +4.5 \\
\hline 49 & $1043+253$ & 104638.6 & +250307 & $210+62$ & 13.1 & 1.8 & 9.72 & 0.93 & 13.0 & +11.0 \\
\hline 50 & $1140+038$ & 114249.1 & +033510 & $265+61$ & 12.0 & 1.0 & 9.18 & 0.68 & 8.0 & +6.7 \\
\hline 51 & $1145-000$ & 114759.7 & -001918 & $271+58$ & 12.4 & 2.8 & 10.01 & 0.91 & 9.0 & +7.5 \\
\hline 52 & $1152-039$ & 115506.1 & -041224 & $277+59$ & 11.5 & 2.2 & 8.43 & 0.91 & 6.0 & +5.0 \\
\hline 53 & $1225+077$ & 122740.4 & +072813 & $285+69$ & 13.1 & 1.7 & 10.85 & 0.68 & 13.0 & +12.0 \\
\hline 54 & $1238-046$ & 124133.2 & -045204 & $298+58$ & 13.1 & 1.2 & 11.07 & 0.75 & 13.0 & +11.0 \\
\hline 55 & $1305+015$ & 130817.7 & +011649 & $313+67$ & 12.2 & 2.3 & 9.81 & 0.81 & 8.0 & +7.5 \\
\hline 56 & $1406+027$ & 140900.7 & +023212 & $343+59$ & 12.8 & 1.0 & 10.39 & 0.74 & 11.0 & +9.5 \\
\hline 57 & $1418-031$ & 142057.2 & -031954 & $341+52$ & 12.0 & 2.5 & 9.51 & 1.00 & 7.5 & +6.0 \\
\hline 58 & $1418+018$ & 142101.2 & +013718 & $347+56$ & 11.8 & 1.9 & 9.13 & 0.86 & 7.0 & +6.0 \\
\hline 59 & $1440+263$ & 144248.4 & +261030 & $037+65$ & 12.4 & 2.6 & 9.06 & 0.90 & 9.0 & +8.0 \\
\hline 60 & $1451+075$ & 145333.7 & +072012 & $004+55$ & 11.7 & 2.1 & 9.38 & 0.97 & 7.0 & +5.5 \\
\hline 61 & $1516+151$ & 151840.2 & +145903 & $021+53$ & 11.1 & 3.8 & 7.34 & 1.45 & 10.0 & +8.0 \\
\hline 62 & $1524+046$ & 152723.6 & +042828 & $009+46$ & 12.0 & 3.9 & 8.13 & 2.03 & 16.0 & +12.0 \\
\hline 63 & $1547+046$ & 154952.6 & +042835 & $013+42$ & 12.2 & 2.4 & 9.79 & 0.75 & 8.0 & +5.5 \\
\hline 64 & $1552-002$ & 155446.0 & -002551 & $008+37$ & 11.3 & 1.8 & 9.15 & 0.78 & 5.0 & +3.0 \\
\hline 65 & $1553+119$ & 155543.6 & +114716 & $022+44$ & 12.4 & 2.0 & 10.56 & 0.74 & 9.0 & +6.0 \\
\hline 66 & $1609-058$ & 161208.6 & -055551 & $006+31$ & 12.3 & 2.1 & 8.75 & 0.97 & 6.0 & +3.0 \\
\hline 67 & $1612+262$ & 161503.4 & +260751 & $043+45$ & 13.1 & 2.2 & 10.90 & 0.80 & 12.0 & +8.5 \\
\hline 68 & $1615-048$ & 161817.1 & -045642 & $008+31$ & 12.6 & 2.3 & 8.63 & 1.23 & 8.0 & +4.0 \\
\hline 69 & $1619+160$ & 162129.4 & +155257 & $031+40$ & 11.8 & 2.5 & 8.85 & 1.09 & 7.0 & +4.5 \\
\hline 70 & $1715+172$ & 171729.3 & +171356 & $039+28$ & 12.2 & 2.7 & 10.03 & 0.75 & 8.0 & +4.0 \\
\hline 71 & $1728+216$ & 173053.7 & +213841 & $044+27$ & 11.1 & 1.9 & 8.14 & 0.95 & 5.0 & +2.5 \\
\hline 72 & $1756+226$ & 175815.6 & +223553 & $048+21$ & 12.0 & 3.7 & 8.07 & 1.44 & 14.0 & +5.0 \\
\hline 73 & $1825+272$ & $1827 \quad 10.2$ & +271440 & $055+17$ & 12.1 & 1.5 & 10.50 & 0.65 & 7.0 & +2.0 \\
\hline 74 & $2029+101$ & 203151.8 & +10 1757 & $054-17$ & 11.8 & 2.1 & 8.69 & 1.01 & 6.0 & -1.8 \\
\hline 75 & $2100+123$ & 210249.9 & +123226 & $061-22$ & 12.7 & 2.4 & 8.72 & 1.18 & 9.5 & -3.5 \\
\hline 76 & $2107+109$ & 210959.0 & +111104 & $061-24$ & 13.2 & 2.4 & 9.01 & 1.40 & 13.0 & -5.5 \\
\hline 77 & $2158+197$ & 220117.5 & +200149 & $077-27$ & 12.5 & 1.5 & 10.29 & 0.63 & 9.0 & -4.0 \\
\hline 78 & $2203+198$ & 220543.8 & +200810 & $078-28$ & 13.2 & 1.9 & 10.31 & 0.87 & 13.0 & -6.0 \\
\hline 79 & $2217+100$ & 221958.5 & +101504 & $073-37$ & 13.3 & 1.8 & 10.25 & 0.80 & 13.0 & -8.0 \\
\hline
\end{tabular}




\section{Appendix A: Limits of the area scanned to find the FBS objects of Table 1}

Table A.1 gives details on the sky regions on which the objects of Table 1 were found. Column (1) is the FBS Zone number, Col. (2) is the area in square degrees, Col. (3) gives the limits in declination in degrees, and Col. (4) provides the limits in right ascension.

\section{Appendix B: Confirmation spectroscopy of FBS car- bon stars}

In order to confirm the carbon-rich nature of FBS objects (including those of Paper I), follow-up slit spectroscopy has been carried out using various intrumental setups. These observations are often performed as backup programs of other projects. Not all FBS objects could be checked, but all observed sources were found to be carbon-rich. The utilised instruments are detailed below.

Spectra from 5700 to $6600 \AA$ were obtained at HauteProvence Observatory with the $1.93-\mathrm{m}$ telescope with the Carelec spectrograph. This instrument was equipped with a 1200 lines $\mathrm{mm}^{-1}$ grating giving a dispersion of $0.45 \AA$ pixel $^{-1}$ on the detector, which was an EEV $2048 \times 1024$ chip, with pixels of $13.5 \mu \mathrm{m}$. The slit was $2^{\prime \prime}$ and the final resolving power is $R \approx 3300$.

Spectra from 5800 to $8400 \AA$ were obtained at the ESO Danish 1.5-m telescope equipped with the DFOSC focal reducer. Grism \# 8 was used to give a dispersion of $1.2 \AA$ pixel $^{-1}$ on the EEV/MAT CCD $2148 \times 4096$ detector. The slit width was $1.5^{\prime \prime}$ and the resolving power is $R \approx 2500$.

Low resolution spectra were taken at the 2.6-m telescope of Byurakan Observatory. The spectrograph had a grating of 600 lines $\mathrm{mm}^{-1}$, providing a dispersion of $1.7 \AA$ pixel $^{-1}$ on a $2063 \times 2058$ CCD detector, with pixels of $15 \mu \mathrm{m}$. The final resolving power is $R \approx 700$.

On medium resolution spectra, molecular bands typical of carbon stars can be seen at 6130 and $6190 \AA$ (due to $\mathrm{C}_{2}$ ), and at 6925 and $7850 \AA$ (due to $\mathrm{CN}$ ). On low resolution spectra, the $\mathrm{C}_{2}$ bands are obvious.

The attached figures concern the following objects:

a) from Paper I, 9 further objects are confirmed (in addition to the 22 objects that had been confirmed in Paper I): FBS $0137+400,0518+687,0644+616,0846-071,0922+786$, 1431-079, 1618-087, 2123-104, 2207-095.
Table A.1. Coordinates of the FBS area surveyed for C stars.

\begin{tabular}{cccc}
\hline $\begin{array}{c}\text { Zone } \\
\text { No. }\end{array}$ & $\begin{array}{c}\text { Area } \\
\mathrm{deg}^{2}\end{array}$ & $\begin{array}{c}\delta \text { limits } \\
\circ\end{array}$ & \multicolumn{2}{c}{$\alpha$ limits } \\
$\mathrm{h} \mathrm{m} \mathrm{h} \mathrm{m}$
\end{tabular}

b) from this paper Table 1, 24 objects are confirmed. At medium resolution: FBS $0318+238,0502+088$, $0520+029,2029+101,2100+123,2107+109,2203+198$, and $2217+100$. At low resolution: FBS $0318+238$, $0502+088,0707+270,0707+310,0729+269,0731+274$, $0826+109,0826+185,0900+034,1043+213,1043+253$, $1225+077,1305+015,1524+253,1524+046,1553+119$, $1756+226,1825+272,2158+197$. 
N. Mauron et al.: FBS and HES carbon stars, Online Material $p 4$
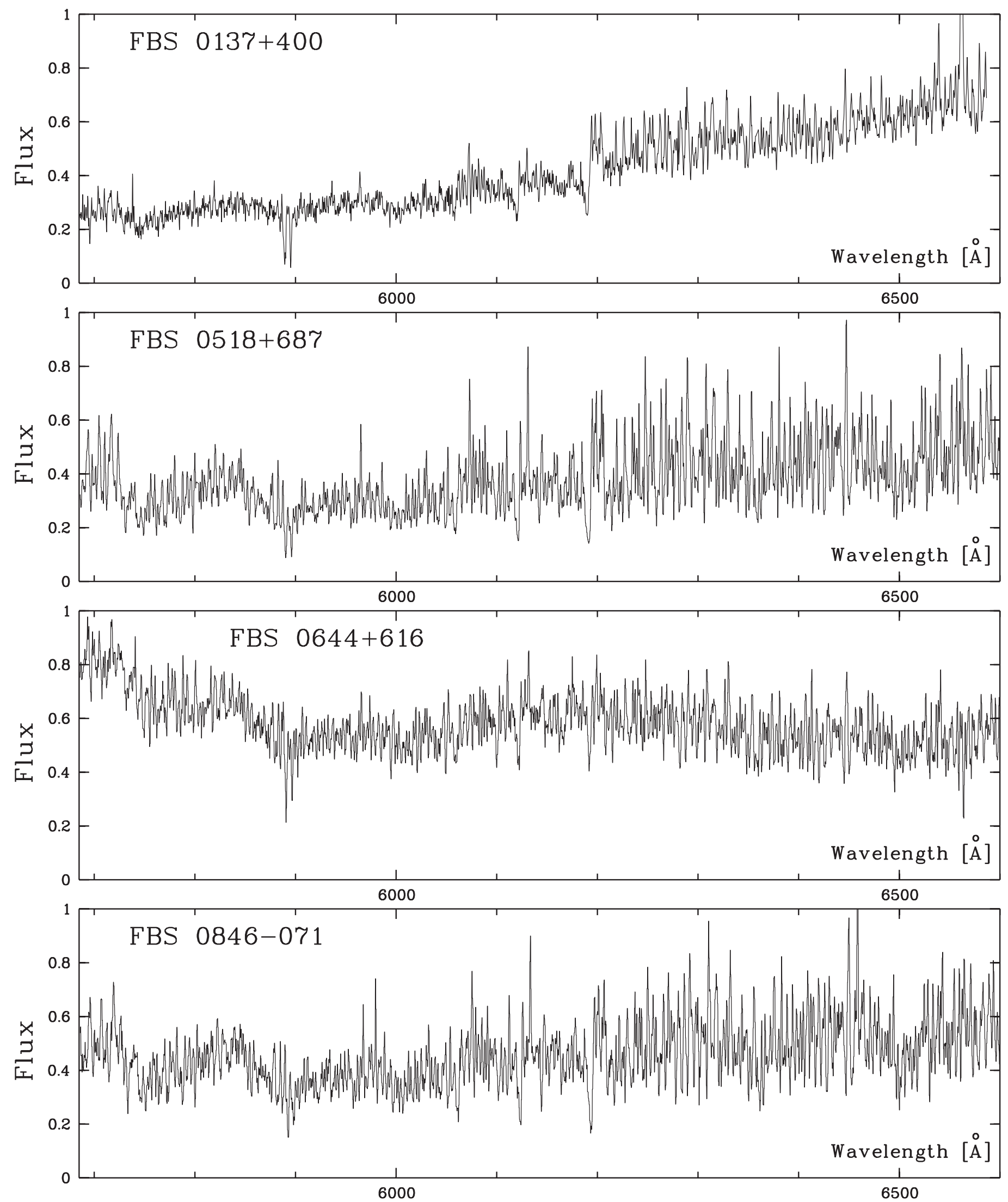
N. Mauron et al.: FBS and HES carbon stars, Online Material p 5
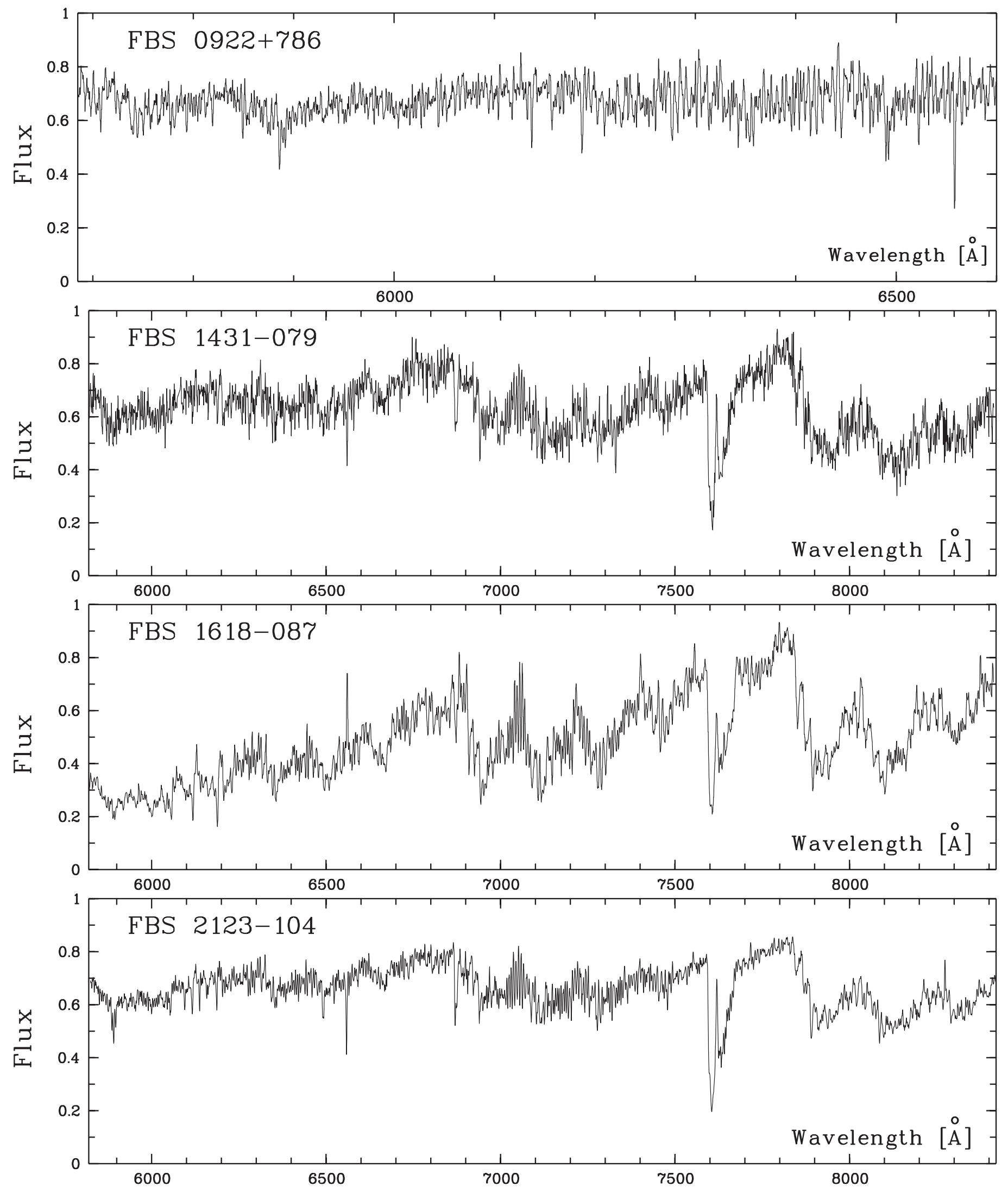
N. Mauron et al.: FBS and HES carbon stars, Online Material p 6
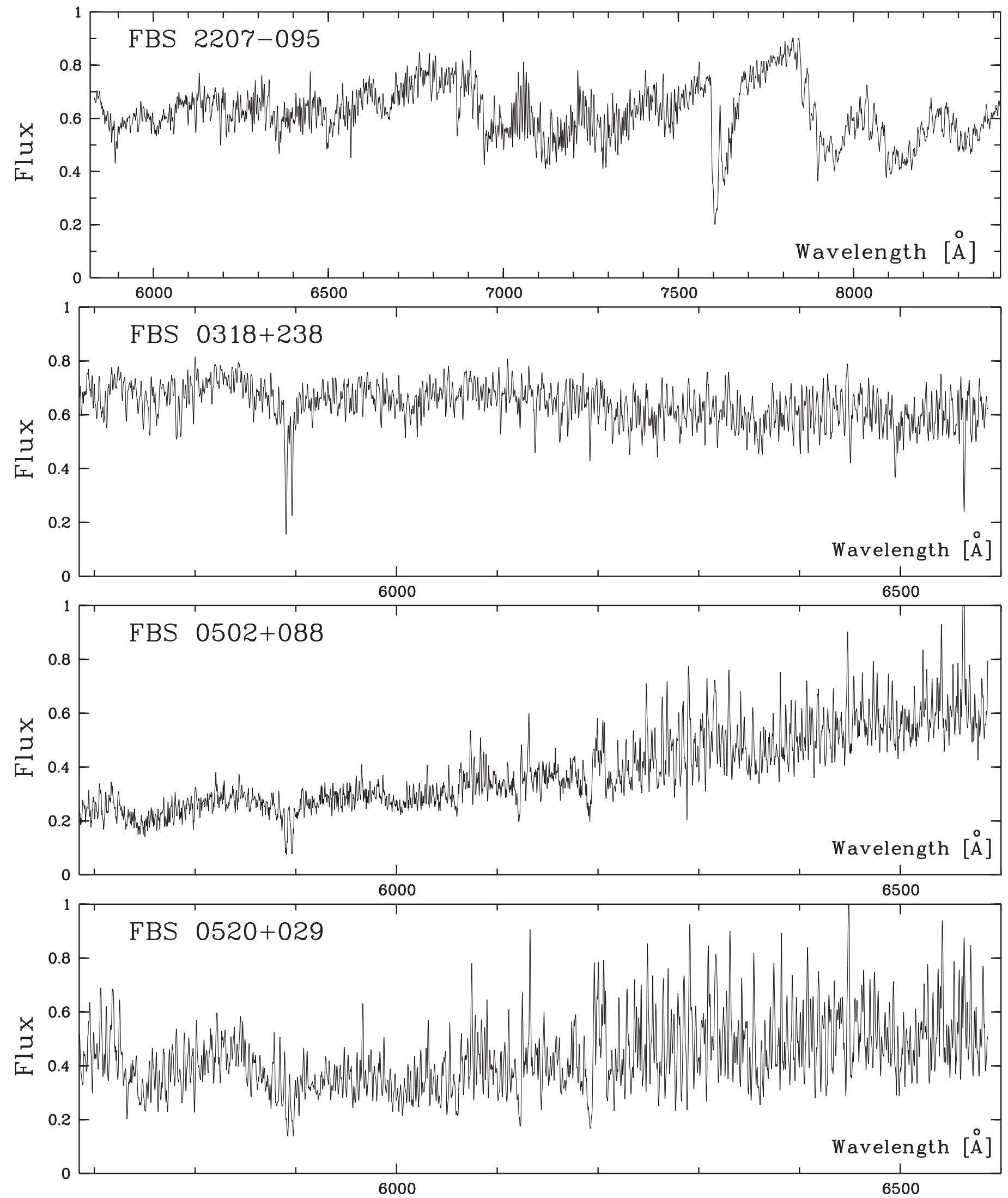
N. Mauron et al.: FBS and HES carbon stars, Online Material $p 7$
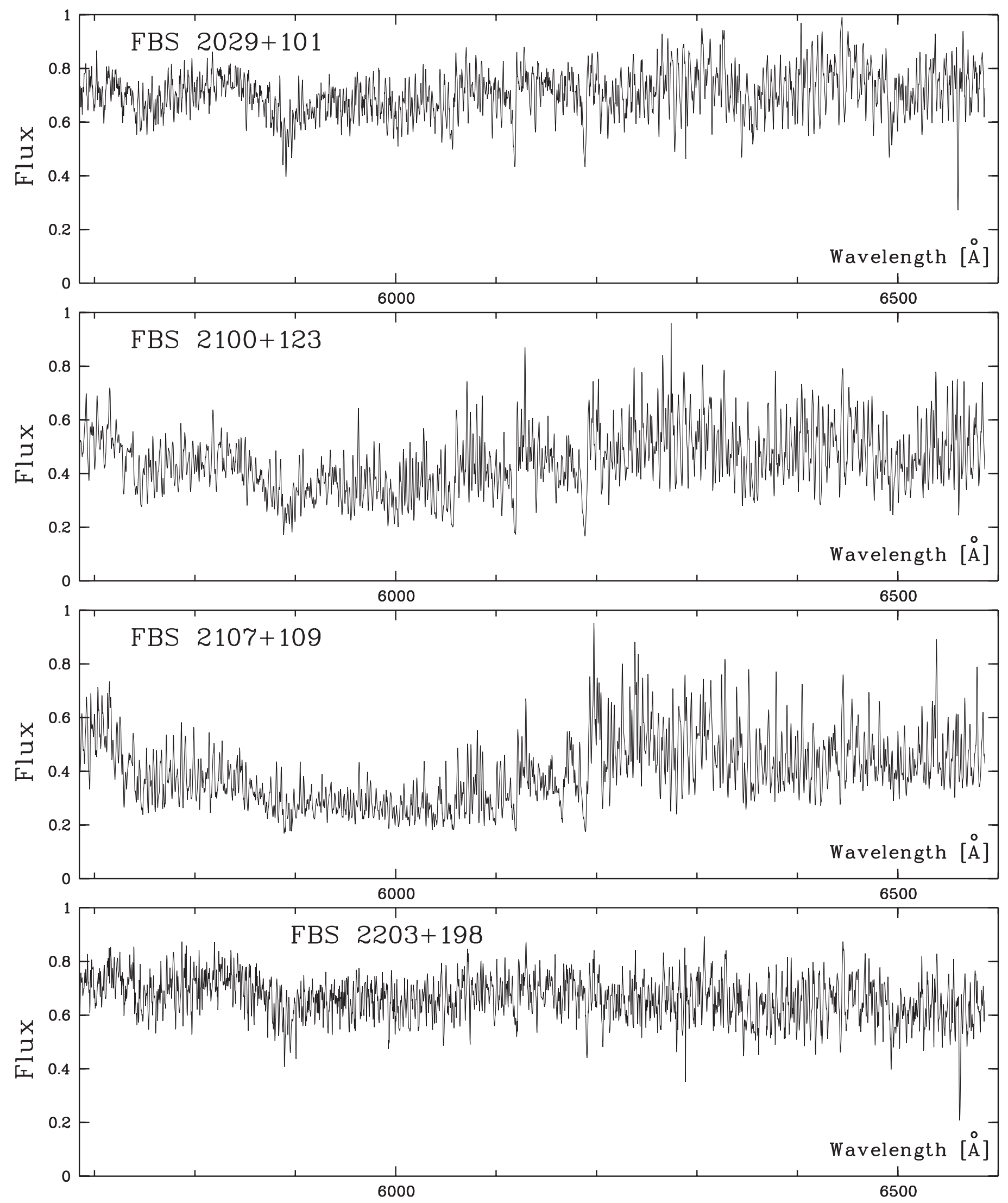
N. Mauron et al.: FBS and HES carbon stars, Online Material $p 8$

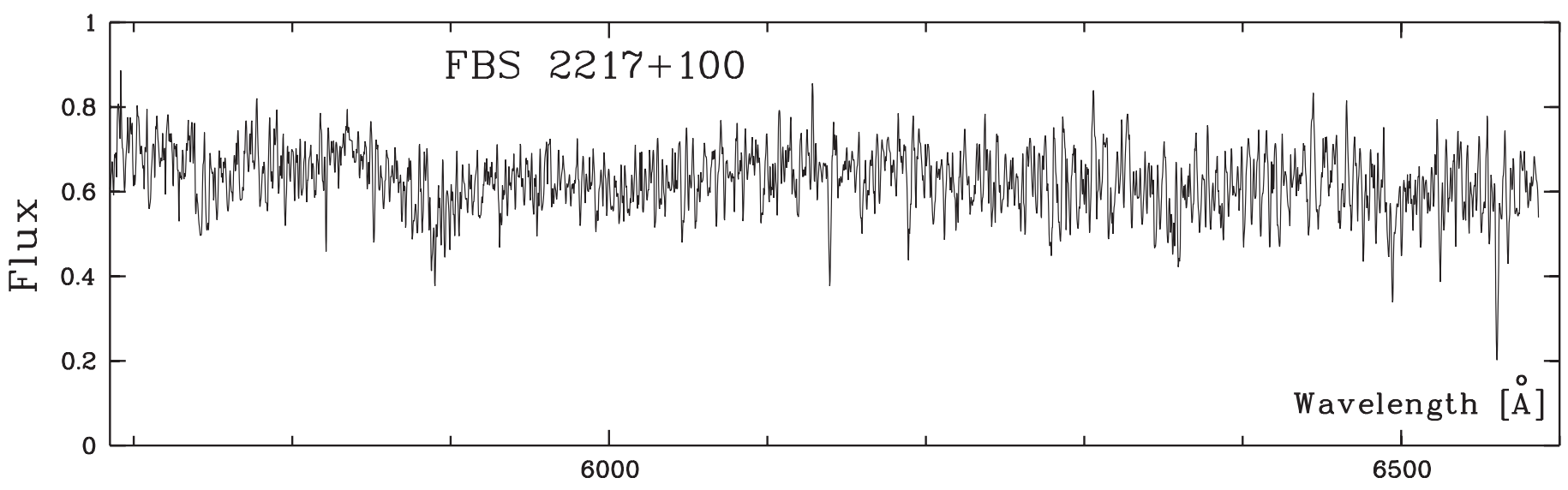


N. Mauron et al.: FBS and HES carbon stars, Online Material $p 9$
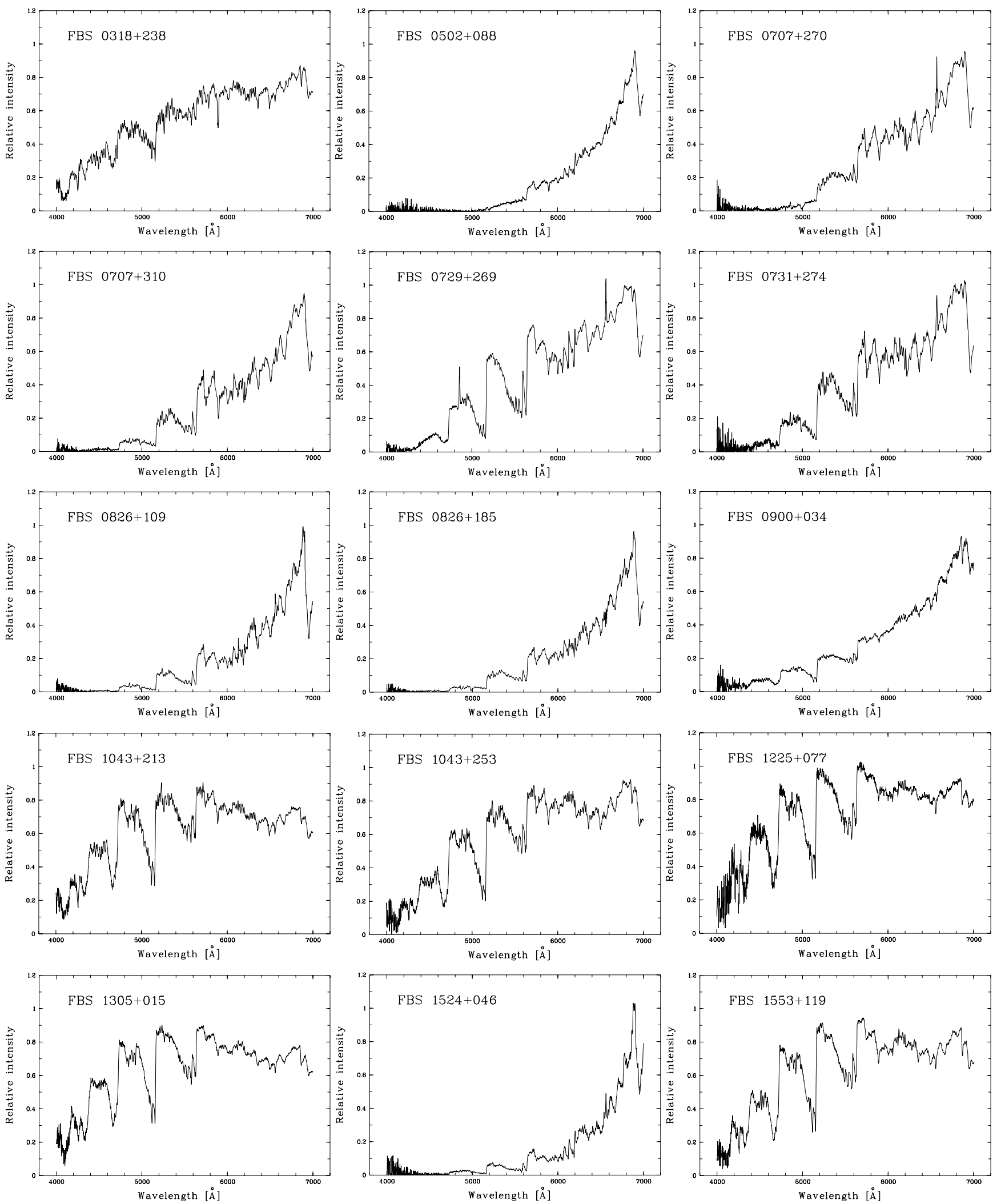
N. Mauron et al.: FBS and HES carbon stars, Online Material p 10
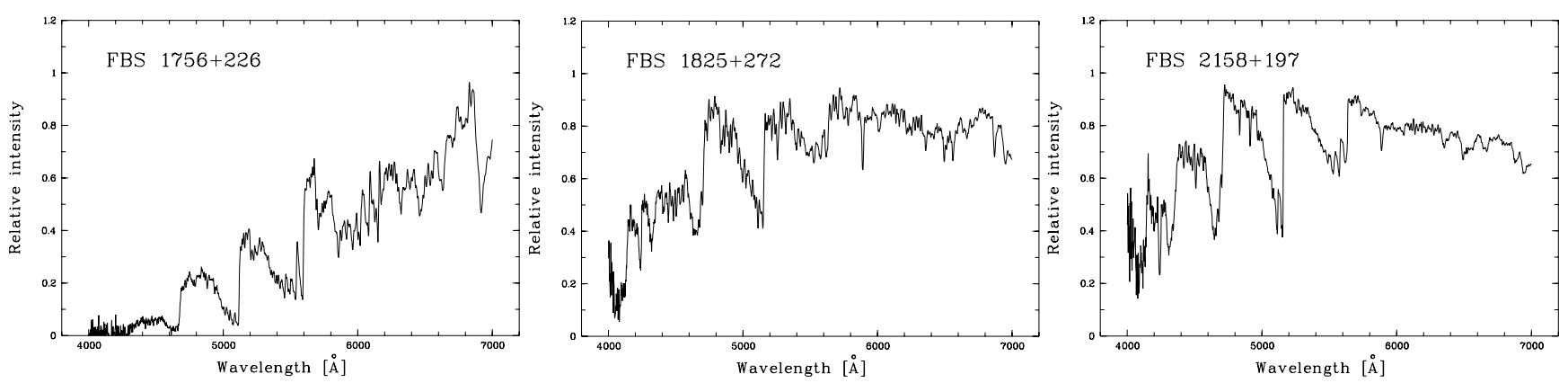